\title{
Relaxed periodic switching controllers of high-frequency DC-DC converters using the $\delta$-operator formulation
}

\author{
Antonio Ventosa-Cutillas, Carolina Albea, Alexandre Seuret and Francisco Gordillo
}

\begin{abstract}
This paper deals with the design of new periodic switching control laws for high frequency DCDC converters. The contributions are twofolds. On a first hand, the DC-DC converter model is rewritten as a periodic switched affine system thanks to a $\delta$ operator formulation, which represents an efficient framework for the numerical discretization at high frequencies. On a second hand, three different control laws are provided, the first one being the usual Lyapunov-based control law and the two others being relaxed versions of this first solution. The benefits of these two new control laws over the usual Lyapunovbased one are demonstrated on a simple example.
\end{abstract}

\section{INTRODUCTION}

Nowadays, there is a relevant interest for DC-DC converters due to their numerous applications in the industry, as for example in computer power supply, cell phones, appliances, automotive, aircraft, etc. These systems can be modeled as switched affine systems (SASs), which represent a particular nonlinear class of switched systems. They correspond to a class of hybrid dynamical systems consisting of several operating modes represented by continuous-time subsystems and a rule that selects between these modes [12]. Compared to the linear case, the affine structure of these systems imposes a set of operating points defined by an averaged dynamic, leading to solutions in the generalized sense of Krasovskii.

Many works found in the literature in continuous time control the SASs by a min-projection strategy [2], [5], [17], even for systems with a general nonlinear form [14], [15]. In these works the provided controllers are good, but may lead to arbitrarily fast switching control. Some solutions to this problem can be found in the literature, as [3], [18], [19], where, the authors aim at ensuring a dwelltime associated with an admissible chattering around the operating point. Nevertheless, [3] does not prove a minimum time associated to the spacial regularization. In [19], a focus on specific electronic architecture related to boost converters is proposed. In addition, the contributions of [18] do not provide a complete stability proof. On the other hand, in [4], the authors present an openloop stabilization strategy based on dwell-time computation, [1] proposes a minimum dwell-time with a space and time regularization, [10] guarantees a minimum and maximum dwell-time by solving optimization problems.

Antonio Ventosa-Cutillas and Francisco Gordillo are with Universidad de Sevilla. Av. Camino de los Descubrimientos s/n. 41092, Sevilla, Spain. aventosa,gordillo@us.es

C. Albea and A. Seuret are with LAAS-CNRS, Univ. de Toulouse, UPS, LAAS, 7 avenue du Colonel Roche, F-31400 Toulouse, France. calbea, aseuret@laas.fr
These solutions present a common characteristic: systems are controlled by aperiodic switching.

In many occasions, it is necessary to control this class of systems with periodic switching, due to physical constraints. In order to deal with this issue, a solution consisting in the discretization of the continuous-time model with a fixed periodic sampling time was provided in [6], [11]. The authors of [6] present a controller based on a Lyapunov function synthesized by solving an optimization problem, whose objective is to minimize the area around the equilibrium, where the solutions converge. On the other hand, in [11], the authors design a sampleddata switching control with an upper-bound designed to ensure robustness in continuous-time systems. Through Linear Matrix Inequalities (LMI) based conditions, the upper-bound of the length of the inter-sampling interval can be directly related to the size of the asymptotic stability set around the considered equilibrium. Practical stability is obtained using Lyapunov-Krasovskii functional and the Jensen's inequality. Both solutions do not consider a high-frequency sampling, beside the fact that they are conservative because the controllers are based on a Lyapunov-function.

In some applications when discretizing these systems, if the sample time is very low, several problems may appear to assess stability, because of numerical issues. Several solutions have been considered in the literature of automatic control. Among them, the $\delta$-operator has been introduced in [16]. It consists of a discretization method that becomes sufficiently close to the continuoustime model, ensuring continuity of the conditions for high-frequency samplings. In [7], a comparison is made between the operator $q$ and the operator $\delta$ to perform this discretization. Here, it is possible to observe as the operator $\delta$ presents as advantage the natural convergence to a continuous system, while avoiding numerical problems when the sampling period is very short. In [20] it is possible to observe that the delta operator is used to perform the sliding mode fuzzy controller of a DC-DC buck converter due to the need for very fast sampling. Therefore, the use of the $\delta$-operator presents a great advantage in the design of controllers with very fast sampling times like the one presented in this paper.

In this paper, we model the DC-DC converter in discrete-time by using the $\delta$-operator and we control the system with a well-known min-projection strategy, based on a Lyapunov function. Then, we propose a periodic-sampling relaxed controller for these systems, allowing to obtain results less conservative, even for highfrequency systems. Moreover, this approach presents a 
trade-off between the sampling period and the size of the chattering effects. Some simulations in Matlab valid our contribution.

The paper is organized as follows: the problem formulation is stated in Section II. Then, a classical controller is presented in Section III. From this, Section IV proposes some relaxed controllers. An optimization of the controllers is given in Section V. Section VI illustrates the potential of this method on a particular DC-DC converter. The paper ends with a conclusion section.

Notation: Throughout the paper $\mathbb{N}$ and $\mathbb{R}$ denote the set of natural and real numbers, respectively. $\mathbb{R}^{n}$ the $\mathrm{n}$ dimensional Euclidean space and $\mathbb{R}^{n \times m}$ the set of all real $n \times m$ matrices. The set composed by the first $N$ positive integers, namely $\{1,2, \ldots, N\}$, is denoted by $\mathbb{K}_{N} . I$ is the identity matrix of suited dimension. The Euclidean norm of vector $x \in \mathbb{R}^{n}$ is denoted by $|x|$. For any symmetric matrix $M$ of $\mathbb{R}^{n \times n}$, the notation $M \succ 0(M \prec 0)$ means that the eigenvalues of $M$ are strictly positive (negative).

\section{Problem Formulation}

\section{A. System data}

Inspired by the work in [6], we focus on the following class of switched affine systems, which is relevant in the context of DC-DC converters

$$
\dot{z}=A_{\sigma} z+a_{\sigma}
$$

where $z \in \mathbb{R}^{n}$ is the state and it is accessible, $A_{\sigma}$ and $a_{\sigma}$ present suited dimensions. The control action is performed through the high frequency switching signal $\sigma \in \mathbb{K}_{N}:=\{1,2, \ldots, N\}$, which may be only modified at sampling instants $t_{k}$, with $k \in \mathbb{N}$. In this paper, the length of the sampling interval $t_{k+1}-t_{k}=T$ is assumed to be constant, known and small enough.

This paper focuses on the design problem of a feedback law for the high frequency periodic switching signal $\sigma$, in such a way to ensure suitable practical convergence properties of the plant state $z$ to a operating equilibrium $z_{e}$, which is not necessarily an equilibrium for the continuous-time dynamics in (1), but can be obtained as an equilibrium for the switching system with arbitrary switching. A necessary and sufficient condition characterizing this equilibrium is then represented by the following standard assumption (see [5], [13]).

Assumption 1: There exists $\lambda=\left[\lambda_{1}, \lambda_{2}, \ldots, \lambda_{N}\right]$ satisfying $\sum_{i \in \mathbb{K}} \lambda_{i}=1$, such that the following convex combination holds:

$$
\sum_{i \in \mathbb{K}} \lambda_{i}\left(A_{i} z_{e}+a_{i}\right)=A(\lambda) z_{e}+a(\lambda)=0 .
$$

Remark 1: It is emphasized that Assumption 1 is both necessary and sufficient for the existence of a suitable switching signal ensuring forward invariance of the point $z_{e}$ (namely inducing an equilibrium at $z_{e}$ ) when understanding solutions in the generalized sense of Krasovskii or Filippov. Indeed, under (2), we can conclude that the error equation of (1):

$$
\dot{x}=A_{\sigma} x+B_{\sigma},
$$

where the error vector is denoted by $x:=z-z_{e}$ and where the matrices $B_{\sigma}$ are defined by $B_{\sigma}:=A_{\sigma} z_{e}+a_{\sigma}$ and verify the following convex combination $\sum_{i \in \mathbb{K}} \lambda_{i} B_{i}=0$. The objective is to ensure that the error state $x$ converges to the equilibrium $x=0$ in the Filippov sense.

In addition, the following property is assumed.

Assumption 2: The matrices $A_{i}$, for $i \in \mathbb{K}_{N}$ are nonsingular and $A(\lambda)$ is Hurwitz.

\section{B. $\delta$-operator for high frequency switching function}

In this paper, we will propose a discrete-time model based on the $\delta$-operator [16], which is suitable for high switching frequencies. The $\delta$-operator has been widely used in the literature to avoid numerical problems in the computation of discrete-time dynamics. This is based on the continuous ones in the situation where the sampling period $T$ is potentially very small. The definition of the $\delta$-operator is as follows. For any function $\xi$ from $\mathbb{R}^{+}$to $\mathbb{R}^{n}$, the vector $\delta \xi_{k}$, at any sampling instant $t_{k} \in \mathbb{R}^{+}$, is defined as follows

$$
\delta \xi_{k}:=\frac{1}{T}\left(\xi_{k+1}-\xi_{k}\right), \quad \forall k \geq 0,
$$

where we used the convention $\xi_{k}=\xi\left(t_{k}\right)$ and $\delta \xi_{k}=$ $\delta \xi\left(t_{k}\right)$, for all integer $k \geq 0$. Hence the dynamics of system (1) can be rewritten in the framework of the $\delta$ operator, which yields the following dynamics

$$
\delta x_{k}=E_{\sigma} x_{k}+F_{\sigma}
$$

where the matrices that defines the system dynamics are given by

$$
E_{\sigma}=\frac{1}{T}\left(e^{A_{\sigma} T}-I\right), \quad F_{\sigma}=\frac{1}{T} \int_{0}^{T} e^{A_{\sigma}(T-s)} d s B_{\sigma} .
$$

The interest of this formulation compared to the usual discrete-time formulation comes from the fact that, when $T$ goes to zero, matrices $E_{\sigma}$ and $F_{\sigma}$ converge to $A_{\sigma}$ and $B_{\sigma}$, respectively. Another important issue is that matrices $E_{\sigma}$ and $F_{\sigma}$ depend explicitly on the switching period $T$. Indeed, considering small values of $T$ may lead to several numerical problems when discretizing (3).

Remark 2: Note that if matrix $A_{\sigma}$ is non singular, then a simple expression of $F_{\sigma}$ is provided by

$$
F_{\sigma}=\frac{e^{A_{\sigma} T}-I}{T} A_{\sigma}^{-1} B_{\sigma}
$$

It is worth noting that model (4) does not account for the continuous evolution of (1) during the intersampling time. It is however possible to characterize the continuous solution by integrating the solution over a sampling interval, leading, for all $t \in\left[t_{k}, t_{k}+T\right]$ and for all $k \in \mathbb{N}$, to

$$
x(t)=e^{A_{\sigma}\left(t-t_{k}\right)} x\left(t_{k}\right)+\int_{t_{k}}^{t} e^{A_{\sigma}\left(\tau-t_{k}\right)} d \tau a_{\sigma}
$$

Since $t$ belongs to the bound interval $\left[t_{k}, t_{k}+T\right]$, the solutions to the system are obviously bounded during the inter sampling time. 


\section{Control objectives}

When considering such switching affine systems, asymptotic stability to zero is in general not possible. Therefore one has to relax the control objectives and to consider attractor sets, which are not necessarily reduced to the equilibrium set. In this paper, we will consider an estimation of a set that ensures that outside $\delta V<0$, which is of the following quadratic form

$$
\mathcal{E}:=\left\{x \in \mathbb{R}^{n}, \quad x^{\top} S x \leq 1\right\} .
$$

with $S$ being a symmetric positive definite matrix to be optimized. This formulation is quite usual and has been used in other contexts as in [1], [6], [11].

This paper focuses on the design problem of a feedback law for the high frequency periodic switching signal $\sigma$, in such a way to ensure suitable practical convergence properties of the plant state $x$ to 0 , which is not necessarily an equilibrium for the continuous-time dynamics in (1), but can be obtained as an equilibrium for the switching system with arbitrary switching. A necessary and sufficient condition characterizing this equilibrium is then represented by the following standard Assumption 2 (see [5], [13]). The problem can be summarized as follows

Problem 1: For any small sampling period $T$, the problem is to find a switching control law that selects, at each sampling time, the mode or subsystem among all possibilities that stabilizes system (1) with certain performance guarantees at its equilibrium.

\section{LYAPUNOV-BASED SWITCHING CONTROL}

Looking at the literature on switched affine systems, one can find the well-known min-projection control law for such a class of systems [6], [11], [17]. The underlying idea of this control law is to select the mode of the system which minimizes the decrease of a quadratic Lyapunov function given by

$$
V(x):=x^{\top} P x, \quad \forall x \in \mathbb{R}^{n}
$$

where $P \succ 0$ is a positive definite matrix of $\mathbb{R}^{n \times n}$. This idea is formalized in the following theorem.

Theorem 1: Consider Assumptions 1 and 2 and matrices $P \succ 0$ and $S \succ 0$ of suited dimension that are solution to the feasibility problem

$$
\Gamma_{i j}^{(1)} \prec 0, \quad \forall i, j \in \mathbb{K}
$$

for any pair $(i, j)$ in $\mathbb{K}^{2}$.

$$
\begin{aligned}
& \Gamma_{i j}^{(1)}=\Psi_{i}(P)+\mu_{i}\left[\begin{array}{cc}
S & 0 \\
0 & -1
\end{array}\right]+\gamma_{i}\left[\Psi_{j}(P)-\Psi_{i}(P)\right], \\
& \Psi_{i}(P)=\left[\begin{array}{cc}
P E_{i}+E_{i}^{\top} P+T E_{i}^{\top} P E_{i} & P F_{i}+T E_{i}^{\top} P F_{i} \\
F_{i}^{\top} P+T F_{i}^{\top} P E_{i} & T F_{i}^{\top} P F_{i}
\end{array}\right],
\end{aligned}
$$

for some given parameters $\gamma_{i}>0$ and $\mu_{i}>0$. Then the switching control (C1) law defined by

$$
\text { (C1) } \sigma\left(x_{k}\right)=\underset{i \in \mathbb{K}_{N}}{\operatorname{argmin}}\left[\begin{array}{c}
x_{k} \\
1
\end{array}\right]^{\top} \Psi_{i}(P)\left[\begin{array}{c}
x_{k} \\
1
\end{array}\right]
$$

guarantees $\delta V<0$ outside of set $\mathcal{E}$.
Proof: Consider the Lyapunov function given in (7), where $P \succ 0 \in \mathbb{R}^{n \times n}$. Let us first compute the expression of $\delta V_{k}$ as follows

$$
\begin{aligned}
\delta V\left(x_{k}\right) & =\frac{1}{T}\left(V\left(x_{k+1}\right)-V\left(x_{k}\right)\right) \\
& =\frac{1}{T}\left(\left(x_{k}+T \delta x_{k}\right)^{\top} P\left(x_{k}+T \delta x_{k}\right)-x_{k}^{\top} P x_{k}\right) \\
& =2 \delta x_{k}^{\top} P x_{k}+T \delta x_{k}^{\top} P \delta x_{k} .
\end{aligned}
$$

Replacing $\delta x_{k}$ by its expression given in (4), and using the definition of the matrix $\Psi_{i}(P)$ provided in (9) yields

$$
\delta V\left(x_{k}\right)=\left[\begin{array}{c}
x_{k} \\
1
\end{array}\right]^{\top} \Psi_{\sigma}(P)\left[\begin{array}{c}
x_{k} \\
1
\end{array}\right] .
$$

Using (9), the previous expression can be rewritten as follows, for any $j$ in $\mathbb{K}$

$$
\begin{aligned}
\delta V\left(x_{k}\right)=\left[\begin{array}{c}
x_{k} \\
1
\end{array}\right]^{\top}\left(\Gamma_{\sigma j}^{(1)}-\mu_{\sigma}\left[\begin{array}{cc}
S & 0 \\
0 & -1
\end{array}\right]\right. \\
\left.\quad-\gamma_{\sigma}\left[\Psi_{j}(P)-\Psi_{\sigma}(P)\right]\right)\left[\begin{array}{c}
x_{k} \\
1
\end{array}\right]
\end{aligned}
$$

Since the matrix inequalities $\Gamma_{i, j}^{(1)} \prec 0$ holds for any pair $(i, j)$ in $\mathbb{K}^{2}$, we have

$$
\begin{aligned}
\delta V\left(x_{k}\right)< & \mu_{\sigma}\left(1-x_{k}^{\top} S x_{k}\right) \\
& -\gamma_{\sigma}\left[\begin{array}{c}
x_{k} \\
1
\end{array}\right]^{\top}\left[\Psi_{j}(P)-\Psi_{\sigma}(P)\right]\left[\begin{array}{c}
x_{k} \\
1
\end{array}\right]
\end{aligned}
$$

Note that the switching control law (10) ensures that the last term of the right hand side of the previous inequality is non positive, which guarantees that

$$
\delta V\left(x_{k}\right)<\mu_{\sigma}\left(1-x_{k}^{\top} S x_{k}\right)
$$

The previous inequality finally guarantees that for any values of $x_{k}$ outside of $\mathcal{E}$ (i.e. $1-x_{k}^{\top} S x_{k}<0$ ), the quantity $\delta V\left(x_{k}\right)$ is strictly negative, which concludes the proof.

\section{RELAXED SWITCHING CONTROLLER}

In the previous section, a Lyapunov-based switching control was presented. This control was clearly inspired from the existing literature on switched affine systems such as [6], [11] but adapted to the $\delta$-operator modelling of DC-DC converters. The motivation of this section is to present a relaxed version of the previous control law. This relaxation considered here is related to the fact that the a priori intuition behind this Lyapunov-based control law might be too restrictive in the sense that the selection of the Lyapunov matrix $P$ is done to verify two distinct purposes, namely, the definition of the Lyapunov function and the construction of the switching signal. Based on this comment, a relaxed control law can be provided by decoupling the two problems of selecting a Lyapunov function and of designing the switching control law.

The relaxed control law is based on the simple idea consisting of keeping the same structure of control law 
presented in (10). However, instead of using the Lyapunov matrix $P$, a new unconstrained matrix is introduced to define the switching law. This is formalized in the following theorem.

Theorem 2: Consider Assumptions 1 and 2, matrices $P \succ 0$ and $S \succ 0$ of suited dimension and a new matrix $N \in \mathbb{R}^{n \times n}$ that are solution to the feasibility problem

$$
\Gamma_{i j}^{(2)} \prec 0, \quad \forall i, j \in \mathbb{K}
$$

where, for any pair $(i, j)$ in $\mathbb{K}^{2}$,

$$
\Gamma_{i j}^{(2)}=\Psi_{i}(P)+\mu_{i}\left[\begin{array}{cc}
S & 0 \\
0 & -1
\end{array}\right]+\gamma_{i}\left[\Psi_{j}(N)-\Psi_{i}(N)\right]
$$

where the matrices $\Psi_{i}(P)$ and $\Psi_{i}(N)$ are given in (9) and, again, for some given parameters $\gamma_{i}>0$ and $\mu_{i}>0$. Then the switching control (C2) law defined by

$$
\text { (C2) } \sigma\left(x_{k}\right)=\underset{i \in \mathbb{K}_{N}}{\operatorname{argmin}}\left[\begin{array}{c}
x_{k} \\
1
\end{array}\right]^{\top} \Psi_{i}(N)\left[\begin{array}{c}
x_{k} \\
1
\end{array}\right]
$$

guarantees $\delta V<0$ outside of set $\mathcal{E}$.

Proof: The proof strictly follows the proof of Theorem 1, except that, now, the switching control law is not characterized by the Lyapunov matrix $P$ but by an arbitrary matrix $N$, which only has to be a solution to the feasibility problem (12).

Remark 3: Compared to Theorem 1, there are two main advantages. The first one relies on the fact that matrix $N$ is not required to be symmetric nor positive. The second one consists in the fact that the switching law is now completely decoupled from the definition of the Lyapunov function. Moreover, one can see that selection $N=P$ in Theorem 2 leads to the same statement as in Theorem 1. This ensures that the set of feasible solutions of (12) is greater than the ones of (8). It is then expected to derive relaxed solutions that will be presented in the example section where the optimization procedure presented later on in Section V is included. $\lrcorner$

\section{Optimisation PROCEDURE}

The feasibility problems proposed in Theorems 1 and 2 , only ensure that there exists a switching control law that stabilizes the system to a bounded region around the equilibrium. Without an optimization process, the resulting regions might be too large to be relevant from the physical point of view. Indeed, considering a too large set $\mathcal{E}$ can possibly increase the chattering effects which are the main phenomena to avoid or limit in the control design of DC-DC converters. This chattering behavior can damage or even break the devices. Therefore, it is necessary to include an optimization procedure in these theorems, whose objective is to minimize the size of the set $\mathcal{E}$. Since this set is fully characterized by the symmetric positive definite matrix $S$, minimizing the size of $\mathcal{E}$ can be achieved by maximizing the determinant of $S$. Based on the discussion above, the following proposition of stated dealing with the optimization of the solutions to the conditions of Theorems 1 and 2 is stated.
Proposition 1: For any a priori fixed scalar parameters $\mu_{j}$ and $\gamma_{i}$, the optimisation problem

$$
\max _{S, P} \operatorname{det}(S), \quad \text { s.t. } \quad \Gamma_{i j}^{(c)} \prec 0, \quad \forall i, j \in \mathbb{K}
$$

for any $c=\{1,2\}$, minimizes the size set (6) guaranteeing $\delta V<0$ outside of set $\mathcal{E}$.

Remark 4: Note that we do not optimize the chattering region, because it is constrained to an ellipse form and, the controller is also constrained for a given structure. Thus, it is expected that set $\mathcal{E}$ will be relaxed with the control given in Theorem 2 , with respect to the control law $\mathrm{C} 1$.

Remark 5: This optimization process strongly depends on the selection of the scalar parameters $\gamma_{i}$ and $\mu_{j}$. Hence it is expected that an iterative procedure to selected the best parameters needs to be included.

Remark 6: Other optimization objectives can be considered such as the maximization of the eigenvalues of $S$, which can be done by maximizing a scalar $\tau$ such that $\tau I \leq S$.

\section{Application to a Boost COnverter}

The control laws introduced above are evaluated on a classical boost converter system. This converter switches at high frequency between two modes $(N=2)$ corresponding to two affine subsystems. The state variable is defined by $x=\left[\begin{array}{ll}i_{L} & v_{c}\end{array}\right]^{\top}$, where $i_{L}$ denotes the inductor current and $v_{c}$ the capacitor voltage.

We take the parameters given in [5] for comparison with the switched control algorithm presented therein, which switches with arbitrary aperiodic switching in the steady-state. This type of switching tends to be complicated in physical applications. The considered nominal values are: $V_{i n}=100 \mathrm{~V}, R=2 \Omega, L=500 \mu \mathrm{H}, C_{o}=$ $470 \mu F$ and $R_{o}=50 \Omega$. The switched system state space model (1) is defined by the following matrices for $i=1,2$ :

$$
A_{i}=\left[\begin{array}{cc}
-\frac{R}{L} & \frac{(1-i)}{L} \\
\frac{(i-1)}{C_{0}} & -\frac{1}{R_{0} C_{0}}
\end{array}\right], \quad a_{i}=\left[\begin{array}{c}
\frac{V_{I N}}{L} \\
0
\end{array}\right] .
$$

The chosen simulation parameters are given by

$$
z_{e}=\left[\begin{array}{ll}
3 & 120
\end{array}\right]^{\top}, \lambda=\left[\begin{array}{ll}
0.22 & 0.78
\end{array}\right]
$$

for which simple calculations ensure the satisfaction of Assumption 2. The optimization problems given in Proposition 1 is solved using the CVX solver [8], [9]. The results obtained with this software illustrate our the efficiency of the new control law presented in Theorem 2 with respect to the Lyapunov-based controller employed in the literature, presented in Theorem 1.

As pointed out in Remark 5, the optimization scheme presented in Proposition 1 delivers different results for different values of $\gamma_{i}$ and $\mu_{j}$. Therefore, a random algorithm has been considered to obtain the best tuple of parameters. The values of these parameters $\mu_{1}, \mu_{2}, \gamma_{1}$ and $\gamma_{2}$, obtained for several values of the sampling period $T_{s}$ are provided in Table I.

Figure 1 shows the state trajectories, set $\mathcal{E}$ and $\delta V>$ 0 surface in the state-plane for the different controllers 


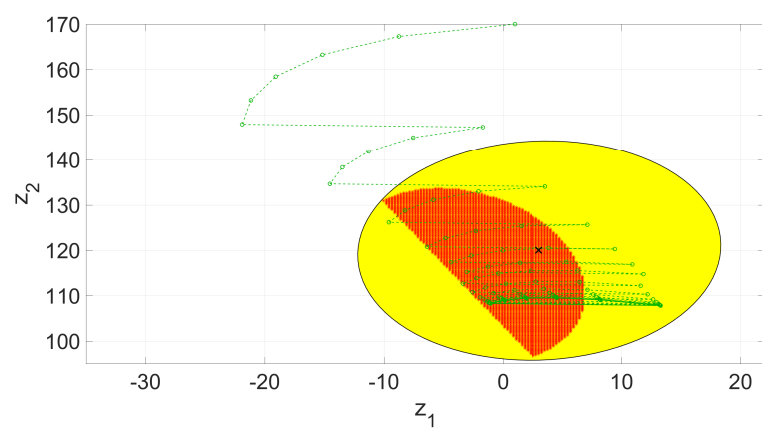

(a) C1 with $T=10^{-4}$.

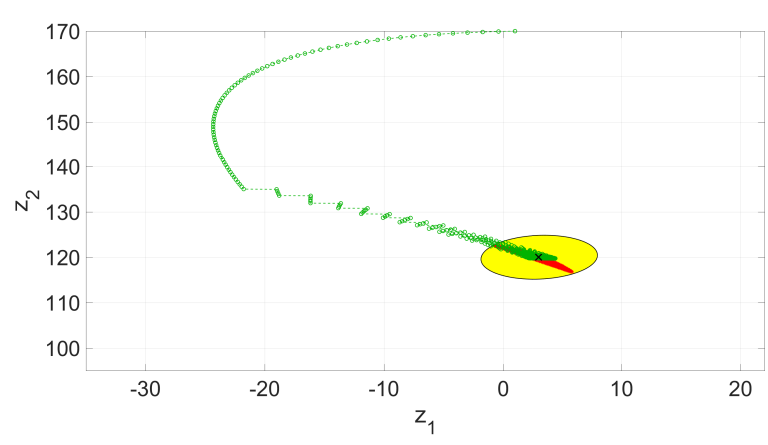

(c) C1 with $T=10^{-5}$.

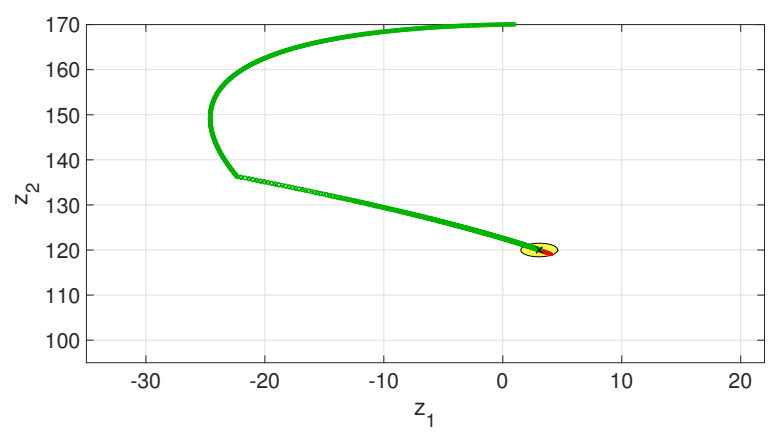

(e) C1 with $T=10^{-6}$;

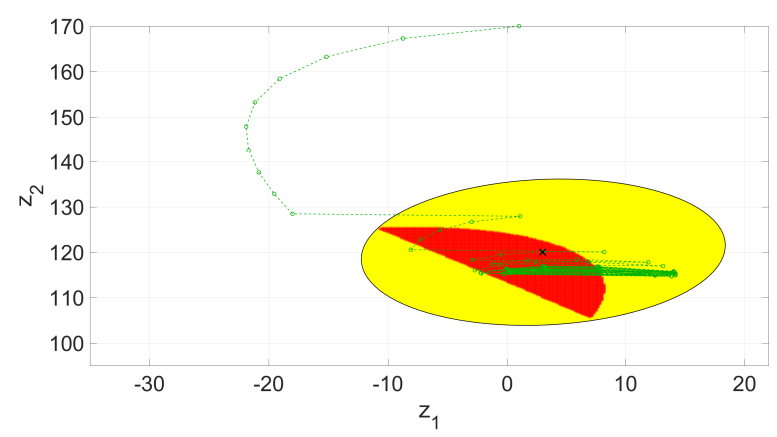

(b) C2 with $T=10^{-4}$.

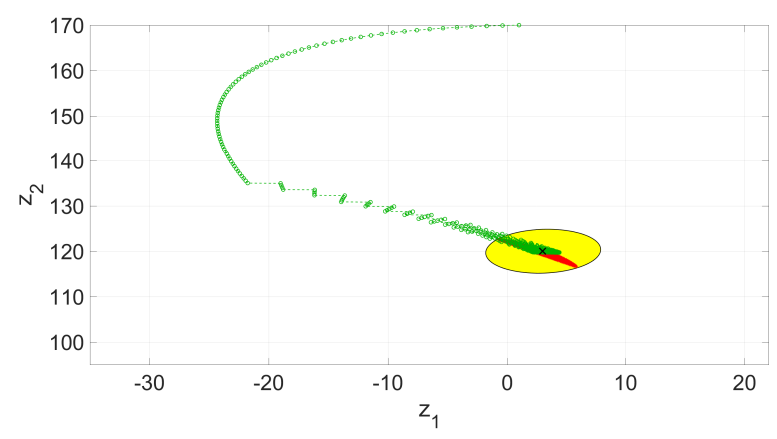

(d) C2 with $T=10^{-5}$.

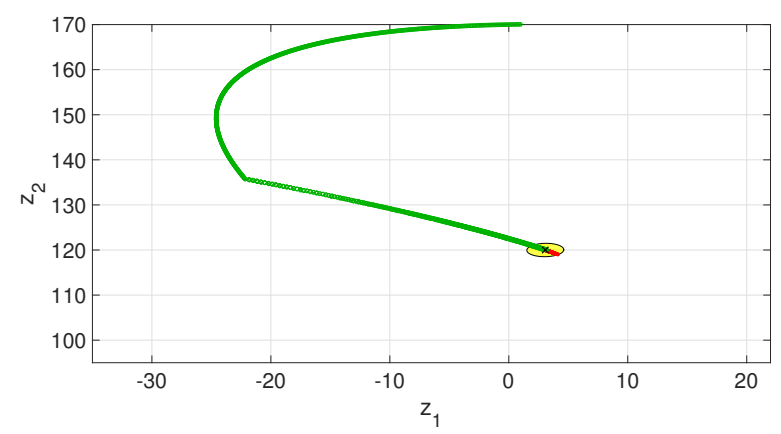

(f) C2 with $T=10^{-6}$.

Fig. 1: Numerical results of Proposition 1. Time trajectories in green, set $\mathcal{E}$ in yellow and $\delta V>0$ in red.

\begin{tabular}{|c|c|c|c|c|c|}
\hline Parameters & $T_{s}$ & $\mu_{1}$ & $\mu_{2}$ & $\gamma_{1}$ & $\gamma_{2}$ \\
\hline \hline Th. 1 & $10^{-4}$ & 9.84 & 9.86 & 0.948 & 0.0515 \\
\hline & $10^{-5}$ & 0.16 & 0.157 & 0.777 & 0.223 \\
\hline & $10^{-6}$ & 0.907 & 0.907 & 0.782 & 0.218 \\
\hline \hline Th. 2 & $10^{-4}$ & 3.16 & 3.16 & 0.916 & 0.327 \\
\hline & $10^{-5}$ & 1 & 1 & 0.349 & 0.1 \\
\hline & $10^{-6}$ & 0.313 & 0.313 & 10 & 2.793 \\
\hline
\end{tabular}

TABLE I: Numerical values of $\mu_{1}, \mu_{2}, \gamma_{1}$ and $\gamma_{2}$.

given in Proposition 1, as well as for different sampling periods. Note that the $\delta V>0$ surface is in the interior of the set $\mathcal{E}$ as is expected from Theorem 1 and 2. Remark also as $\mathcal{E}$ is reduced, as $T_{s}$ decreases. We can see that set $\mathcal{E}$ is reduced with control law $\mathrm{C} 2$ w.r.t. $\mathrm{C} 1$, showing as the control law $\mathrm{C} 2$ provide reduced region with respect to
C1. This is consistent with Remark 3. Another important remark concerns the fact that the region where $\delta V>0$ is concentrated in a smaller area in control law $\mathrm{C} 2$ with respect to $\mathrm{C} 1$.

These simulations demonstrate the advantages of the relaxed control law over the existing Lyapunov-based one. Indeed, our proposed controller C2 allow to control efficiently the DC-DC converters under study even with relatively high sampling period with a notable reduction of the switches of these systems, which ensures an increase of the lifespan and the reduction of the dissipated energy.

\section{ViI. Conclusions}

In this paper, we have presented two main contributions. The first one deals with the definition of accurate 
discrete-time model for high frequency sampling DCDC converters. The second contribution consists in the extension of the usual Lyapunov-based control laws employed in this domain to a less restrictive control law that encompass this first formulation. This new control law delivers notable improvements with respect to the Lyapunov-based control law in terms when comparing the estimate of the set $\mathcal{E}$ of the switched affine system.

\section{ACKNOWLEDGMENTS}

This work has been funded under grant MINECOFEDER DPI2016-75294-C2-1-R.

\section{REFERENCES}

[1] C. Albea, G. Garcia, S. Hadjeras, M.W.P.M.H. Heemels, and L. Zaccarian. Practical stabilisation of switched an systems with dwell-time guarantees. Submitted to IEEE Trans. on Automatic Control, 2017.

[2] C. Albea, G. Garcia, and L. Zaccarian. Hybrid dynamic modeling and control of switched affine systems: application to DC-DC converters. In IEEE Conference on Decision and Control, Osaka, Japan, 2015.

[3] J. Buisson, P.Y. Richard, and H. Cormerais. On the stabilisation of switching electrical power converters. In Hybrid Systems: Computation and Control, volume 3414 of Lecture Notes in Computer Science, pages 184-197. Springer Berlin Heidelberg, 2005.

[4] P. Colaneri, J. C. Geromel, and A. Astolfi. Stabilization of continuous-time switched nonlinear systems. Systems \& 6 Control Letters, 57(1):95-103, 2008.

[5] G. S. Deaecto, J. C. Geromel, F.S. Garcia, and J.A. Pomilio. Switched affine systems control design with application to DCDC converters. IET control theory \& applications, 4(7):1201$1210,2010$.

[6] G.S. Deaecto, M. Souza, and J.C. Geromel. Discrete-time switched linear systems state feedback design with application to networked control. IEEE Trans. on Automatic Control, 60(3):877-881, 2015.

[7] B. L. Eidson, John Y. Hung, and R. M. Nelms. In In Proc. IEEE South East conference, pages 1-6.

[8] M. Grant and S. Boyd. Graph implementations for nonsmooth convex programs. In V. Blondel, S. Boyd, and H. Kimura, editors, Recent Advances in Learning and Control, Lecture Notes in Control and Information Sciences, pages 95-110. Springer-Verlag Limited, 2008.

[9] M. Grant and S. Boyd. CVX: Matlab software for disciplined convex programming, version 2.1, mar 2014.

[10] P. Hauroigne, P. Riedinger, and C. Iung. Switched affine systems using sampled-data controllers: Robust and guaranteed stabilisation. IEEE Trans. on Automatic Control, 56(12):2929-2935, 2011.

[11] L. Hetel and E. Fridman. Robust sampled-data control of switched affine systems. IEEE Trans. on Automatic Control, 58(11):2922-2928, 2013.

[12] D. Liberzon and A.S. Morse. Basic problems in stability and design of switched systems. IEEE Control Systems Magazine, 19(5):59-70, 1999.

[13] D. Liberzon and A.S. Morse. Basic problems in stability and design of switched systems. IEEE Control Systems Magazine, 19(5):59-70, 1999.

[14] J. Liu, X. Liu, and W.C. Xie. On the (h0, h)-stabilization of switched nonlinear systems via state-dependent switching rule. Applied Mathematics and Computation, 217(5):20672083, 2010.

[15] Y. Lu and W. Zhang. A piecewise smooth control-lyapunov function framework for switching stabilization. Automatica, $76: 258-265,2017$.

[16] R.H. Middleton and G.C. Goodwin. Improved Finite Word Length Characteristics in Digital Control Using Delta Operators. IEEE Trans. on Automatic Control, 31(11):1015-1021, 1986.

[17] S. Pettersson and B. Lennartson. Stabilization of hybrid systems using a min-projection strategy. In Proc. of the 2001 IEEE American Control Conference, 2001., volume 1, pages 223-228.
[18] M. Senesky, G. Eirea, and T.J. Koo. Hybrid modelling and control of power electronics. In Hybrid Systems: Computation and Control, volume 2623 of Lecture Notes in Computer Science, pages 450-465. Springer Berlin Heidelberg, 2003.

[19] T. A.F. Theunisse, J. Chai, R.G. Sanfelice, and W.P. Heemels. Robust global stabilization of the dc-dc boost converter via hybrid control. IEEE Trans. on Circuits and Systems I: Regular Papers, 62(4):1052-1061, 2015.

[20] K. Viji, A. Kumar, and R. Nagaraj. Improved Delta Operator based Discrete Sliding Mode Fuzzy Controller for Buck Converter. Indian Journal of Science and Technology, 10(25):1-11, 2017. 